\title{
EXPERIMENTAL MODEL
}

\section{A resource for selecting animal models of heart disease}

\author{
Federspiel, J.D. et al. PLoS Biol. 17, e3000437 (2019)
}

The use of animal models recapitulating human condition is essential to understand the mechanisms underlying cardiac disease. Mice and pigs have been extensively used in cardiovascular research, but fundamental differences between these animal models and humans, including differences in cardiac protein expression, limit their translational value. A new study presents the cardiac proteomes of four animals with dual circulatory systems - the pig, Sus scrofa; the mouse, Mus musculus; and two frogs, Xenopus laevis and Xenopus tropicalis - and compares their protein profiles with that of humans. This new resource might help researchers find the right model to understand the function of specific proteins in cardiac disease.

The different cardiac proteomes were analysed by liquid chromatography coupled with tandem mass spectrometry (LCMS/MS) and mapped to human protein sequences. Out of 4,710 quantifiable proteins, 1,770 were found in all four vertebrates, demonstrating the existence of a conserved core of proteins involved in important cardiac functions such as metabolism, translation and cellular organization. The analysis also uncovered mammalian-specific and amphibian-specific protein profiles, as well as sets of proteins that are unique for each species.

Proteins often carry out their functions as part of larger protein complexes. Therefore, the investigators used a computational approach to quantify the abundance of protein complexes in each species. Proteins identified in the LC-MS/MS analysis were mapped to two databases of human complexes: the comprehensive resource of mammalian protein complexes (CORUM) and the cardiac BioSNAP database, which is based on experimental evidence from physical human protein-protein interaction. Similar to the protein analysis, the comparison of the different profiles for protein complexes revealed shared clusters and others that were species-specific, reflecting the variation across the cardiac proteomes of these models.

Further analysis of the data showed that the mammalian proteome is enriched in proteins involved in metabolic processes related to lipid and carbohydrate

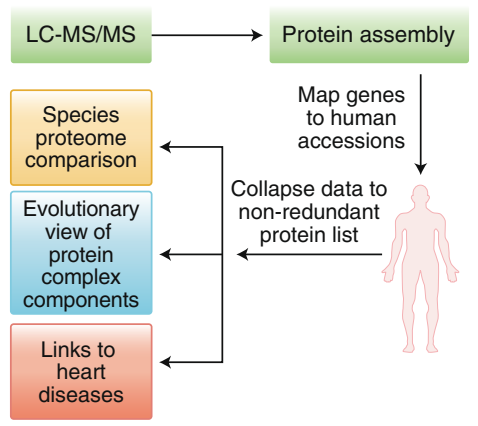

Workflow for investigating multispecies cardiac proteomes. Reproduced from Federspiel et al. (2019) PLOS Publications.

metabolism, whereas the Xenopus proteome is enriched in cell-cycle associated proteins, intracellular signal transduction and cell communication proteins. The mouse heart is specifically enriched in proteins involved in vesicle-mediated transport functions, whereas the pig heart is enriched in proteins with mitochondrial and transcriptional functions; $X$. tropicalis and $X$. laevis express a high number of RNA processing and cell adhesion proteins, respectively. "Altogether, our results highlight the existence of differential abundance profiles for protein complexes within these cardiac model systems, which may drive some of the different observed phenotypes in these species," say the investigators in their report.

Next the team investigated the correlation between human proteins that have been previously linked to cardiac disease and the cardiac proteome of each species. They found that some of these human disease-causing proteins showed speciesspecific abundances. For example, dilated cardiomyopathy, the most common type of nonischemic cardiomyopathy, can be caused in humans by mutations in the genes encoding cardiac phospholamban (PLN), delta-sarcoglycan (SGCD) and RNA-binding protein 20 (RBM20). Here, RBM20 was detected only in the mouse heart, whereas PLN and SGCD were both enriched in the pig and mouse hearts. Atrioventricular septal disease is a heart defect caused in humans by mutations in CRELD1, Gap junction alpha-1 (GJA1), transcription factor GATA-4 (GATA4), and GATA6; by looking at their dataset, the investigators found that all four proteins were differentially expressed across the models. "Together, these findings lead us to propose that the capacity of an animal model to accurately mimic the pathologies of a given heart disease may be directly related to the abundance level of those proteins in that species," explain the investigators.

To further validate the usefulness of their dataset, they decided to investigate the role of Kcp, a cysteine-rich protein expressed in humans that was only detected in frog samples. They used TALEN gene-editing technology to introduce a loss-of-function mutation in the Kcp locus of X. laevis. Froglets that were homozygous for the mutation showed a decrease in blood flow at stage 64, indicative of poor cardiac function, and died before stage 66 . Contrast-CT imaging revealed that mutant froglets presented with valve defects, leading the investigators to hypothesize that $K c p$ null froglets were dying from myxomatosous degeneration of the atrioventricular valve, a condition observed in humans. Xenopus is already a well-established model for studying development, because it allows easy imaging and the use of high-throughput omics approaches. The identification of human cardiac proteins specifically enriched in the frog versus other species now makes Xenopus a promising non-mammalian alternative system for modeling protein function with potential translational relevance to the human heart.

"Our findings, and the resources we make available for further study, will enable researchers to determine the most appropriate model systems for exploring the molecular mechanisms for specific cellular events of cardiac development and disease," conclude the investigators in the discussion of the study. The mass spectrometry proteomics data have been deposited to the ProteomeXchange Consortium.

Alexandra Le Bras

Published online: 14 October 2019

https://doi.org/10.1038/s41684-019-0425-4 